\title{
Paradoxical response with miliary pulmonary nodules in a patient with tuberculous pericarditis
}

\author{
Li-Ta Keng MD, Lih-Yu Chang MD \\ — Cite as: CMAJ 2019 May 6;191:E506. doi: 10.1503/cmaj.181330
}

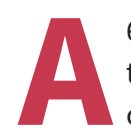

62-year-old woman with hypertension and chronic, inactive hepatitis $B$ infection presented with a 1-month history of fever, exertional dyspnea and weight loss. Chest radiography and computed tomography (CT) showed massive pericardial effusion without pulmonary infiltrates (Figure $1 \mathrm{~A}$ and $\mathrm{B}$ ). A culture of the effusion grew pan-susceptible Mycobacterium tuberculosis, and we diagnosed tuberculous pericarditis. Testing for HIV was negative. We provided standard antituberculosis treatment under direct observation without missed doses. After 3 months of treatment, our patient was symptom-free, but follow-up chest radiography and CT showed newly developed miliary nodules in her lungs, distributed randomly, even though the pericardial effusion had improved (Figure $1 \mathrm{C}$ and D). A transbronchial biopsy showed noncaseating granulomatous inflammation without acid-fast bacteria (Appendix 1, available at www.cmaj.ca/lookup/suppl/ doi:10.1503/cmaj.181330/-/DC1). Nucleic acid amplification test, acid-fast stain, and fungal and mycobacterial culture of bronchoalveolar lavage and lung biopsy specimens were all negative. There was no evidence of tuberculous re-exposure, and we diagnosed a paradoxical response. Because our patient was asymptomatic, we did not prescribe antiinflammatory medications. Follow-up chest radiography and $\mathrm{CT}$ at the end of the 9-month treatment period showed complete resolution of miliary pulmonary nodules.

The term "paradoxical response" refers to an inflammatory reaction associated with antituberculosis treatment. It is characterized by the clinical or radiologic worsening of pre-existing lesions or development of new lesions after initial improvement, in the absence of disease relapse or another diagnosis. Most reported cases manifest as fever or the worsening of extrapulmonary lesions (e.g., lymph nodes, pleura or brain). ${ }^{1}$ It occurs more often in patients with HIV infection or in whom tumour necrosis factor blockade was discontinued because of active tuberculosis. ${ }^{2}$ Paradoxical response is rarely reported in patients with tuberculous pericarditis, and cases with miliary pulmonary nodules are rare. Because paradoxical response can be clinically indistinguishable from treatment failure, it remains a diagnosis of exclusion. ${ }^{3}$

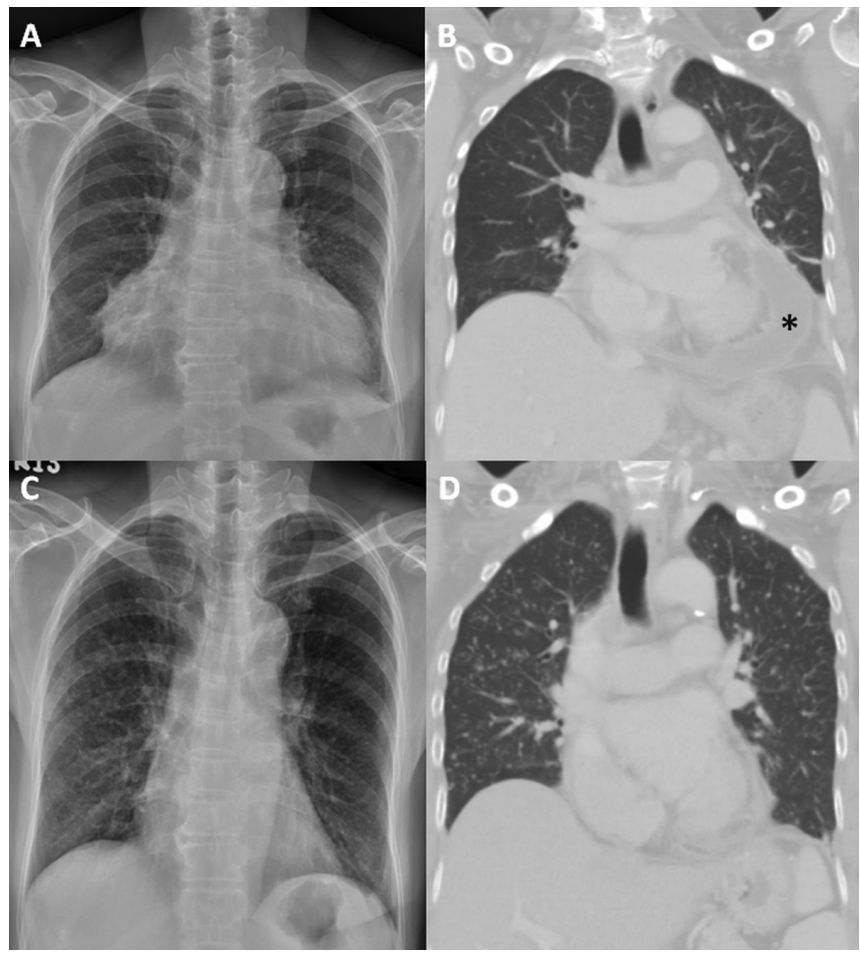

Figure 1: Chest radiograph (A) and computed tomography scan (B) in a 62-yearold woman with tuberculous pericarditis, showing enlarged cardiac silhouette with massive pericardial effusion (asterisk) and no pulmonary infiltrates. Chest radiograph (C) and computed tomography scan (D) 3 months after antituberculosis treatment showing newly developed miliary nodules with random distribution bilaterally in the lungs, and decreased pericardial effusion.

\section{References}

1. Brown CS, Smith CJ, Breen RA, et al. Determinants of treatment-related paradoxical reactions during anti-tuberculosis therapy: a case control study. $B M C$ Infect Dis 2016;16:479.

2. Watanabe S, Kaneko Y, Kawamoto $\mathrm{H}$, et al. Paradoxical response with increased tumor necrosis factor- $\alpha$ levels to anti-tuberculosis treatment in a patient with disseminated tuberculosis. Respir Med Case Rep 2017;20:201-4.

3. Meintjes G, Rangaka MX, Maartens G, et al. Novel relationship between tuberculosis immune reconstitution inflammatory syndrome and antitubercular drug resistance. Clin Infect Dis 2009;48:667-76.
Competing interests: None declared.

This article has been peer reviewed.

The authors have obtained patient consent.
Affiliation: Department of Internal Medicine, National Taiwan University Hospital Hsin-Chu Branch, Hsin-Chu, Taiwan

Correspondence to: Li-Ta Keng, ltkeng@gmail.com 